group-individual variation. Concern over what the other girls might think tended to parallel the amount of agreement actually produced under group-oriented conditions. While this result is consistent with our hypothesis that gredie concem would yield higher levels of agreement, the effect is relatively weak.

Consistent with earlier findings, Table 1 shows that $\mathrm{Ss}^{\prime}$ task confidence (Item 5) and perceived task difficulty (Item 3) varied predictably and significantly as a function of prior agreement. Task confidence decreased under both orientations as the level of prior agreement diminished, and perceived task difficulty increased directly as a function of prior agreement. Thus, these findings corroborated the hypothesis of the Julian, Regula. \& Hollander (1968) study of the mediating role that task confidence plays in determining the effect which the level of prior agreement has on subsequent willingness to agree. Ratings under the comparable individually oriented conditions again showed that following $100 \%$ agreement, the task was seen as distinctly less difficult than any of the partial agreement conditions. This result confirmed again the "over-confidence" that apparently leads to the dramatic increase in subsequent agreement following $100 \%$ prior agreement.

\section{Post-Session Ratings}

The postsession questionnaire reflected the complex combined effects of the independent conditions and the influence phase of the experiment and. therefore, is difficult to interpret unambiguously. The greater enjoyment and attraction of group-oriented conditions shown on Items 1 and 7 of the intervening questionnaire were reaffirmed on the postsession questionnaire, again with no differential effects of levels of prior group agreement. Strong effects of the prior agreement conditions were revealed on the postsession ratings of perceived disagreement and perceived influence. Quite veridically, Ss said they agreed more with the others in Phase 2 of the $100 \%$ prior agreement condition and agreed less following the lower levels of prior agreement. Veridical perceptions were also indicated by the ratings of perceived influence under the $100 \%$ and $75 \%$ conditions. However, there was minimal perceived influence attributed to the others judgments under the $25 \%$ agreement condition, although, as Fig. 1 shows, there were relatively higher levels of agreement there.

This study attempted to demonstrate the exchange of agreement under minimal group conditions, with only partial success. In his recent review, Nord (1969) has suggested that two things are necessary for exchange: a medium and something that is to be gamed in the exchange. In the present paradigm, the assumption has been made that "agreement" functions as such a medium for exchange-that agreement from others is a desirable commodity for which there is a demand. Given a medium of exchange in both orientations, then, the next question can be phrased: What is gained by the exchange under group-oriented rather than individually oriented conditions? It would appear that a critical factor in maintaining exchange processes as stressed by Hollander \& Willis (1967) and more recently by Pruitt (1968) is the anticipation of future reward from the exchange. Recognition of this factor implies that the tendency to reciprocate agreement under circumstances such as those studied here does not depend so much on the agreement previously supplied by the others, but rather on whether continued agreement with those others is seen to build the potential for future rewards. Although in the present study, Ss were led to believe there would be a number of sets of group judgments, they knew that they had been recruited for only $1 \mathrm{~h}$ to participate in groups which essentially had no future. If this observation is correct. then it suggests the necessity of focusing on natural or long-term groups in any test of exchange processes.

$$
\text { RHEH REVICIS }
$$

HOLLANDFR. F. P.. JLLIAN, J. W., \& HAALAND, G. A. Conformity process and prior group support. Journal of Personality \& Social Psycholog:. 1965, 6. 852.858.

HOLL ANDER. F. P., \& WILLIS, R. H. Some current issues in the psychology of conformity and nonconformity. Psychological Bulletin, 1967, 68, 62-76.

JONES, E. E., \& GERARD. H. B. Foundations of social psychology: New York: Wiley, 1967.

JLLIAN. J. W., REGLLA, C. R., \& HOLLANDER. E. P. Effects of prior agreement by others on task confidence and conformity. Journal of Personality \& Social Psychology. 1968, 9, 171-178.

JLLIAN, J. W.. RYCKMAN, R. M., \& HOLLANDER. E. P. Effects of prior group support on conformity. Journal of Social Psychology. 1969. 77, 189-196.

NORD, W. R. Social exchange theory: An integrative approach to social conformity. Psychological Bulletin, 1969, 71, 174-208.

PRLITT. D. G. Reciprocity and credit building in a laboratory dyad. Joumal of Personality \& Social Psychology, 1968. 8, 143-147. NOTE

1. The $F$ value for the interaction of level of agreement with orientation was less than 1.00 .

\title{
Scaling apparent distance in a natural outdoor setting
}

\author{
ROBERT TEGHTSOONIAN and MARTHA TEGHTSOONIAN* \\ Smith College, Northampton, Mass. 01060
}

Judgments of apparent distance in outdoor settings are power functions of physical distance, just as they have been shown to be in indoor settings. But the exponents obtained out of doors are not only appreciably lower-all less than 1.00-but are affected by range: the exponent is largest for the smallest ratio of extreme distances.

There is evidence that apparent distance judged in natural indoor settings is an accelerating function of physical distance. Kunnapas (1960) and Teghtsoonian \& Teghtsoonian (1969) have obtained judgments of indoor distances which are power functions of physical distance, the exponents ranging from 1.15 to 1.47 . On the other hand, distance judgments in outdoor settings show no such acceleration: E. J. Gibson and her associates (1954, 1955), Gilinsky (1951), Harway (1963), and Luria, Kinney, \& Weissman (1967) have found apparent

*We thank Eleanor Oldach. Judy Gibbons, and Ellen Dibble. who helped run $S$ s and tabulate data. outdoor distance to be nearly linear with physical distance or a decelerating function of it.

But these two groups of studies differ in at least two respects other than setting. First, ranges used out of doors tend to be larger than those used indoors, and these larger ranges, rather than any intrinsic characteristic of the setting, may account for the difference in exponents. Second, direct comparison of the two groups of studies is hampered by the wide variety of scaling techniques represented. Therefore, it seemed desirable to apply the methods of our study of indoor distances (Teghtsoonian \& Teghtsoonian, 1969) to an outdoor setting, employing ranges comparable to those previously studied 
Table 1

Distance Ranges* and Exponents for Outdoor and Indoor Settings

\begin{tabular}{|c|c|c|c|c|c|c|c|}
\hline \multicolumn{4}{|c|}{ Outdoor } & \multicolumn{4}{|c|}{ Indoor** } \\
\hline \multicolumn{2}{|c|}{ Distance* (Feet) } & \multicolumn{2}{|c|}{ Exponent } & \multicolumn{2}{|c|}{ Distance $^{*}($ Feet $)$} & \multicolumn{2}{|c|}{ Exponent } \\
\hline $\begin{array}{l}\text { Maxi- } \\
\text { mumi }\end{array}$ & $\begin{array}{c}\text { Ratio } \\
\text { Max/Min }\end{array}$ & Mean & SD & $\begin{array}{l}\text { Maxi- } \\
\text { mum }\end{array}$ & $\begin{array}{c}\text { Ratio } \\
\text { Max/Min } \\
\end{array}$ & Mean & $S D$ \\
\hline 37 & 7.4 & .99 & .13 & 45 & 9.0 & 1.15 & .20 \\
\hline 110 & 22.0 & .89 & .13 & 80 & 16.0 & 1.26 & .37 \\
\hline 480 & 96.0 & .85 & .13 & & & & \\
\hline
\end{tabular}

* Minimum distance was 5 ft for all ranges.

**Data from Teghtsoonian \& Teghtsoonian (1969).

both indoors and outdoors. Accordingly, we obtained magnitude estimations of the apparent distance of targets located in the same outdoor setting but distributed over three different ranges, from $5 \mathrm{ft}$ to 37 , 110 , and $480 \mathrm{ft}$.

\section{SUBJECTS}

The Ss were 48 high school students, 22 male and 26 female. They were recruited from summer courses and through newspaper advertisements and were paid for their participation.

\section{MATERIALS}

The viewing situation was an expanse of grass, closely mowed, smooth and without exceptional features. There were trees and bushes at the sides and the far end. Its visually bounded width, while variable, was about $700 \mathrm{ft}$; its visually bounded length was about $1,200 \mathrm{ft}$, although an abrupt drop in ground level occurred about $700 \mathrm{ft}$ from $\mathrm{S}$.

Three different distance ranges were studied: Range 37 was $5-37 \mathrm{ft}$; Range 110 , $5.110 \mathrm{ft}$ : and Range $480,5-480 \mathrm{ft}$. Each range contained nine target distances, spaced at approximately equal logarithmic intervals. The distance markers were nine plywood rectangles, all 6 in. wide and varying in height from 14 to 34 in.; each had two short wooden legs which fitted into wire holders in the ground. The inconspicuous wire holders remained in place throughout the experiment, while only one marker was placed at a time.

\section{PROCEDURE}

The Ss were run in groups as they were available--one to seven at a time, with a modal value of two. As nearly as possible, four Ss were run on a given range, then the next four Ss on another range, and so on. A different presentation order, with a random pairing of markers to target distances, was used for each set of four Ss.

The method of free-modulus magnitude estimation was used. Ss were read the instructions, which emphasized the use of whatever numbers seemed appropriate to represent apparent distances; they were told that physical distances might or might not be the same as apparent distances. and that it was how the distances looked that was important. As a marker was placed, each $S$ in turn stepped up to a designated spot, looked, wrote down a judgment, and turned away. E placed the markers for the six near distances and signaled $\mathrm{S}$ when to make judgments; an assistant placed the markers for the three far distances.

\section{RESULTS AND DISCUSSION}

For each $S$ a straight line was fitted by the method of least squares, relating log magnitude estimation to $\log$ distance. The mean slopes of these lines are $0.99,0.89$, and 0.85 for Ranges 37, 110, and 480, respectively; these means--which are estimates of the corresponding power law exponents-together with their standard deviations, are shown in Table 1 . The good quality of the fits for individual $S s$ is indicated by the mean coefficients of determination, $r^{2}$, for individual fitted lines-.984, .983, and .974 for Ranges 37 , 110, and 480, respectively-and for grouped data by the coefficient of determination for the mean regression line-over .99 for all three ranges. These results are consistent with those previously obtained in outdoor settings; apparent distance is a linear or decelerating function of physical distance.

Comparing these results with those from our previous study of indoor distances (also shown in. Table 1), we find that setting may, indeed, have an effect on the exponent of the power function. With method identical and range comparable, indoor settings generate higher exponents for the apparent distance function. This difference may reflect certain characteristics which differentiate natural settings indoors from those out of doors. One possibility is the greater visual differentiation of indoor space; generally, there are more objects and a greater variety of patterns and textures indoors than out. It may be that apparent filled distance grows increasingly more rapidly as a function of physical distance than does apparent empty distance. A second differentiating characteristic is the greater maximum visible distance out of doors; generally one can see much further out of doors than in. Perhaps it is this difference which is responsible for the lower exponents for outdoor distances.

There is an effect of range on mean exponent in these data. A one-way analysis of variance indicated a reliable range effect $(\mathrm{F}=4.78, \mathrm{df}=2.45: .01<\mathrm{p}<.05)$, which subsequent tests by the Newman-Keuls method showed to be due to the shortest range condition. The exponents decline as the ratio of the extreme distances used increases. Our data support Künnapas's hypothesis (1960) of such an effect for apparent distance. But his estimate of the magnitude of the effect is probably distorted by his inclusion of a low exponent derived from Gilinsky's outdoor data (1951) along with his own higher exponents obtained for an indoor setting. If setting is indeed a parameter of the apparent distance exponent and is independent of range effects, the latter can be estimated accurately only by variation within a single setting. The absence of such a range effect in our earlier study of indoor distances may have been due to the selection of ranges that were too similar to produce a reliable effect. But it is also possible that the confounding of range with setting (two different rooms, differing in length, for the two ranges) in that study served to conceal the effects of range.

To summarize, further evidence has been provided that apparent distance is a power function of physical distance. There is also support for the inference drawn from earlier studies that the exponents obtained for distance judgments in outdoor settings are systematically lower than those for distance judgments in indoor settings. Finally, the power-law exponents for apparent distance may decline when larger stimulus ranges are employed.

\section{REFERENCES}

GIBSON, E. J., \& BERGMAN, R. The effect of training on absolute estimation of distance over the ground. Journal of Experimental Psychology, 1954, 48, 473-482.

GIBSON, E. J., BERGMAN, R., \& PURDY, J. The effect of prior training with a scale of distance on absolute and relative judgments of distance over ground. Journal of Experimental Psychology, 1955, 50, 97-105.

GILINSKY, A. S. Perceived size and distance in visual space. Psychological Review, 1951, 58, 460-482.

HARWAY, N. I. Judgments of distance in children and adults. Joumal of Experimental Psychology, 1963, 65. 385-390.

KÜNNAPAS, T. Scales for subjective distance. Scandinavian Journal of Psychology, 1960, 1 . 187-192.

LURIA, S. M., KINNEY, J. A. S., \& WEISSMAN, $S$. Estimates of size and distance undervater. American Journal of Psychology, 1967, 80, 282-286.

TEGHTSOONIAN, M., \& TEGHTSOONIAN, R. Scaling apparent distance in natural indoor settings. Psychonomic Science, 1969, 16. 281-283. 